\title{
ALTERAÇÕES QUÍMICAS E AVALIAÇÃO DE P DISPONÍVEL NA REGIÃO ADJACENTE AOS GRÂNULOS DE SUPERFOSFATO TRIPLO E DIAMÔNIO FOSFATO EM SOLOS ÁCIDOS ${ }^{(1)}$
}

\author{
Jaqueline Pereira Machado de Oliveira ${ }^{(2)}$, Paulo Roberto Ernani ${ }^{(3)}$, Luciano Colpo \\ Gatiboni $^{(3)}$ \& Angélica Pegoraro ${ }^{(4)}$
}

\section{RESUMO}

\begin{abstract}
O P tem baixa mobilidade no solo resultando em altas concentrações nas regiões adjacentes aos grânulos de fertilizantes fosfatados. Isso pode interferir na disponibilidade de $\mathrm{P}$ e na composição química do solo nesses locais. $\mathrm{O}$ objetivo deste estudo foi avaliar a composição química do solo e a mobilidade de $\mathbf{P}$ nas adjacências de regiões fertilizadas com fosfatos de amônio ou de cálcio, em solos ácidos. Este trabalho foi realizado em 2009, com amostras de dois solos catarinenses. Os tratamentos consistiram de combinações de superfosfato triplo (SFT) ou de fosfato diamônico (DAP) com $\mathrm{KCl}$, além de um controle somente com $\mathrm{KCl}$. As unidades experimentais foram constituídas por colunas de PVC com $10,0 \mathrm{~cm}$ de diâmetro e 12,0 cm de comprimento, preenchidas com $1,60 \mathrm{~kg}$ de solo (base úmida). Foram aplicadas quantidades equivalentes a $200 \mathrm{~kg} \mathrm{ha}^{-1}$ de $\mathrm{P}_{2} \mathrm{O}_{5}$ e de $\mathrm{K}_{2} \mathrm{O}$, considerando a área de contato superficial de solo de $10^{4} \mathrm{~m}^{2}$. Tentando simular a aplicação localizada de fertilizantes, essas quantidades foram estipuladas para contatarem com $357 \mathrm{~m}^{2}$ de solo, tendo sido aplicadas 10,$47 ; 9,56$; e 7,33 g por coluna de SFT, DAP e KCl respectivamente, numa área de contato de $0,00785 \mathrm{~m}^{2}$. Quarenta e cinco dias após a aplicação dos fertilizantes, as colunas foram desmontadas e o solo foi analisado a cada centímetro distante do local de aplicação dos grânulos. $O$ $P$ foi quantificado pelos métodos Mehlich-1 e da resina trocadora de ânions (RTA), numa sequencia de nove extrações cumulativas. O DAP aumentou o pH e diminuiu o Al nas adjacências dos grânulos, porém o SFT aumentou Ca e Mg. A quantidade cumulativa extraída de $\mathbf{P}$ variou com o tipo de adubo fosfatado, a distância dos grânulos, o tipo de solo e o método de extração. Na camada distante até $1,0 \mathrm{~cm}$ dos adubos, o SFT proporcionou os maiores valores de $P$, que chegaram a atingir
\end{abstract}

\footnotetext{
(1) Parte da Tese de Doutorado do primeiro autor. Trabalho financiado pelo CNPq. Recebido para publicação em 3 de junho de 2013 e aprovado em 17 de julho de 2014.

(2) Pós-doutoranda PNPD Capes Institucional, Universidade Tecnológica Federal do Paraná - UIFPR-DV. Estrada Boa Esperança km 4. CEP 85.660-000 Dois Vizinhos (PR). E-mail: jaqpmachado@gmail.com

(3) Professor Adjunto, Centro de Ciências Agroveterinárias - CCA, UDESC. Bolsista de Produtividade em Pesquisa do CNPq. Email: prernani@cav.udesc.br, gatiboni@udesc.br

(4) Engenheira Agrônoma. Av. São Paulo, 335. CEP 79560-000 Chapadão do Sul (MS). E-mail: angélica.pegoraro@monsanto.com
} 


\begin{abstract}
$10.276 \mathrm{mg} \mathrm{dm}^{-3}$; nas duas camadas seguintes $(1,0-2,0 \mathrm{e} 2,0-3,0 \mathrm{~cm})$, isso normalmente ocorreu com o DAP. O Mehlich-1 quantificou sempre mais $P$ do que a RTA nas adjacências dos fertilizantes fosfatados. A movimentação do $P$ nos dois solos ocorreu até o quarto centímetro distante do local de aplicação dos adubos, e a composição química nessas adjacências variou com o fertilizante fosfatado, o tipo de solo, o método de extração e a distância dos grânulos, mas necessita ser mais bem estudada.
\end{abstract}

Termos de indexação: fluxo difusivo, cátions trocáveis, reações do fósforo, Mehlich-1, resina trocadora de ânions.

\title{
SUMMARY: CHEMICAL CHANGES AND EVALUATION OF AVAILABLE P NEAR THE GRANULES OF TRIPLE SUPERPHOSPHATE AND DIAMMONIUM PHOSPHATE IN ACID SOILS
}

\begin{abstract}
Phosphorus has low mobility in the soil. Thus, its concentration near the granules of phosphate fertilizers in the soil is very high, which may affect P availability and the soil chemical composition in these regions. This study was carried out to evaluate the soil chemical composition near the granules of calcium or ammonium phosphates and P mobility in two Brazilian acid soils. The experiment was carried out in 2009 and treatments consisted of a combination of triple superphosphate (TSP) or diammonium phosphate (DAP) with KCl, in addition to a control with only $\mathrm{KCl}$. Each experimental unit was constituted by PVC columns (12 cm long and $10 \mathrm{~cm}$ in diameter), subsequently filled with $1.20 \mathrm{~kg}$ of soil (dry base). The amount of TSP, DAP and KCl applied to each experimental unit was 10.47, 9.56 and $7.33 \mathrm{~g}$ respectively, which, considering a soil volume of $2 \times 10^{3} \mathrm{~m}^{3}$, would be equivalent to $200 \mathrm{~kg} \mathrm{ha}^{-1}$ of $\mathrm{P}_{2} \mathrm{O}_{5}$ and $200 \mathrm{~kg} \mathrm{ha} \mathrm{g}^{-1}$ of $\mathrm{K}_{2} \mathrm{O}$. Forty-five days after fertilizers application the soil volume in each centimeter distant from the granules was chemically quantified. For $P$, nine consecutive extractions were performed using the methods of Mehlich-1 and anion exchangeable resin (AER). DAP increased $p H$ and decreased exchangeable Al near the granules while SFT increased both, Ca and Mg. The amount of cumulative P extracted varied with phosphate fertilizers, distance from the granules, soil type and extraction method. In the 0 to $1.0 \mathrm{~cm}$ layer distant from the granules TSP gave the highest Pvalues, that reached up to 10,276 $\mathrm{mg} \mathrm{dm}^{-3}$; in the two following layers (1.0 to 2,0 and 2.0 to $3.0 \mathrm{~cm}$ ), this occurred with DAP. Mehlich-1 always quantified more P than AER in the soil near the phosphate fertilizers. The movement of P in both soils occurred until the fourth centimeter away from the granules and the soil chemical composition in these regions varied with the phosphate fertilizer, soil type, extraction method and distance from the granules, which needs to be better studied.
\end{abstract}

Index terms: diffusive flux, exchangeable cations, phosphorus reactions, Mehlich 1, anion exchangeable resin.

\section{INTRODUÇÃO}

O fósforo do solo disponível às plantas é constituído pelo $\mathrm{P}$ orgânico, pelo $\mathrm{P}$ adsorvido aos coloides minerais e pelo $\mathrm{P}$ precipitado, principalmente com $\mathrm{Al}, \mathrm{Fe}$ e Ca. Para tornar-se disponível às plantas, o $\mathrm{P}$ orgânico tem que ser mineralizado pelos microrganismos; o $\mathrm{P}$ adsorvido tem que ser liberado dos "sítios" de adsorção; e os precipitados de P precisam ser dissolvidos. Após estar na solução do solo, o P necessita migrar até as raízes para só então ser absorvido. A restrição em qualquer dessas duas etapas (liberação para a solução do solo e movimentação até as raízes) limita a absorção do nutriente pelas plantas (Stevenson \& Cole, 1999).

A movimentação de $\mathrm{P}$ no solo é realizada predominantemente pelo mecanismo de difusão
(Barber, 1962), o qual depende do teor de água (Kelly et al., 1997), do tamanho e da distribuição de poros (Dierolf et al., 1997), da concentração iônica da solução do solo (Oliveira et al., 2004), do pH (Ernani et al., 2002), do poder tampão de $P$ e principalmente da concentração de P na solução do solo (Ernani et al., 2004; Neves et al., 2009). Essa, por sua vez, depende das reações que ocorrem nas regiões adjacentes aos grânulos de fertilizantes. Como o P se movimenta poucos milímetros no solo durante uma estação de crescimento vegetal, tanto a concentração quanto sua reposição na solução nas proximidades dos adubos fosfatados desempenham papel fundamental na disponibilidade de $\mathrm{P}$ às plantas.

Após a dissolução de qualquer fertilizante fosfatado no solo, ocorrem inúmeras reações nas adjacências dos grânulos, sendo algumas estritamente 
relacionadas com o $\mathrm{P}$, principalmente precipitação e adsorção específica, e outras relacionadas com atributos do solo, principalmente alterações no $\mathrm{pH}$, na densidade de cargas elétricas e na condutividade elétrica; todas variam com o tipo de fosfato adicionado. Enquanto os fosfatos de cálcio e o monoamônio fosfato diminuem o pH nas adjacências dos grânulos, o diamônio fosfato proporciona efeito oposto (Ernani et al., 2002; Mantovani et al., 2007). A modificação no $\mathrm{pH}$ dessas microrregiões pode influenciar na atividade do $\mathrm{Al}$ em solos muito ácidos e, consequentemente, na sua fitotoxicidade. Alterações no $\mathrm{pH}$ do solo também influenciam a formação e dissolução dos precipitados de P: em $\mathrm{pH}$ baixo, predomina a formação de fosfatos de $\mathrm{Fe}$ e Al; em valores de $\mathrm{pH}$ mais elevados, os fosfatos de Ca. Nas regiões adjacentes aos grânulos dos adubos fosfatados, entretanto, a magnitude dessas reações não tem sido quantificada.

Considerando as diferentes formas em que o $\mathrm{P}$ se encontra no solo, os métodos analíticos que quantificam o $\mathrm{P}$ disponível às plantas estão divididos em grupos. No Brasil, utilizam-se extratores que agem por dissolução ácida e por troca iônica. $\mathrm{O}$ método de Mehlich-1 extrai parte do P dos coloides inorgânicos por dissolução ácida e quantifica, em ordem crescente de efetividade, as formas precipitadas com $\mathrm{Ca}, \mathrm{Al}$ e Fe. As principais vantagens desse método são a facilidade de execução e o baixo custo analítico; no entanto, tem-se observado que esse apresenta alguns problemas na estimativa dos teores de $\mathrm{P}$ no solo, como a exaustão da capacidade de extração com o aumento do teor de argila e do grau de intemperização do solo, além de superestimar a disponibilidade de $\mathrm{P}$ em solos previamente adubados com fosfatos naturais (Gatiboni et al., 2002; 2003).

O método que utiliza a resina trocadora de ânions (RTA) extrai o P do solo por troca iônica, onde o P migra dos sítios de adsorção dos coloides para as cargas positivas existentes na resina. Esse método não apresenta extração preferencial de $\mathrm{P}$ com relação aos tipos de coloides do solo, porém há controvérsias com relação à sua capacidade de estimar o fator quantidade de P. Silva \& Raij (1999) consideraram a RTA melhor que os outros extratores de $\mathrm{P}$, em virtude do processo de extração assemelhar-se à ação das raízes das plantas, da inexistência de perda de força de extração em solos com alto teor de argila e de superestimação do $\mathrm{P}$ disponível em solos que receberam adubação com fosfatos naturais. Rheinheimer et al. (2003), entretanto, verificaram que esse método é sensível à textura do solo, pois extraiu mais $\mathrm{P}$ do que o Mehlich-1 nos com altos teores de argila, menos naqueles de texturas leves.

Este estudo foi conduzido com o objetivo de avaliar a composição química do solo ao redor de grânulos de fertilizantes fosfatados por dois métodos extratores, assim como a mobilidade de nutrientes, a partir da adição de fosfatos de amônio ou de cálcio, juntos com cloreto de potássio, em dois solos ácidos.

\section{MATERIAL E MÉTODOS}

O experimento foi realizado em laboratório, na Faculdade de Agronomia da Universidade do Estado de Santa Catarina (UDESC), em Lages, SC, em 2009. Utilizaram-se amostras de um Cambissolo Húmico (CH) e de um Nitossolo Vermelho (NV), coletadas na camada de $0-20 \mathrm{~cm}$ de áreas nativas nunca anteriormente cultivadas ou fertilizadas. A escolha desses solos foi com base nas suas composições químicas (Quadro 1). Posteriormente à coleta, os solos foram passados em peneira com abertura de malha de $0,4 \mathrm{~cm}$.

As unidades experimentais foram constituídas por colunas de PVC com 10,0 cm de diâmetro e 12,0 cm de comprimento. Cada coluna foi montada pela justaposição de anéis concêntricos com as seguintes alturas: o superior tinha $2,0 \mathrm{~cm}$; os cinco seguintes tinham $1,0 \mathrm{~cm}$ cada; e o anel mais inferior tinha $5,0 \mathrm{~cm}$. As amostras de solo foram colocadas somente nos $10 \mathrm{~cm}$ inferiores de cada coluna.

Os tratamentos consistiram de combinações de superfosfato triplo (SFT) ou de diamônio fosfato (DAP) com cloreto de potássio $(\mathrm{KCl})$, além de um controle com apenas $\mathrm{KCl}$. Os grânulos de cada fertilizante, todos com diâmetro entre 0,5 e 2,0 mm, foram distribuídos uniformemente entre duas folhas de papel-filtro (whatman 42), alocadas na superfície de junção do primeiro com o segundo anel, a partir da extremidade superior, perfazendo uma área de contato dos fertilizantes com o solo de $0,00785 \mathrm{~m}^{2}$ de cada lado. As doses de cada fertilizante foram determinadas considerando a aplicação de $200 \mathrm{~kg}$ de $\mathrm{P}_{2} \mathrm{O}_{5}$ e de $\mathrm{K}_{2} \mathrm{O}$, espalhados uniformemente a uma área superficial $10^{4} \mathrm{~m}^{2}$. Quando os adubos são aplicados em linhas nas lavouras, esses entram em contato com menores volumes de solo, relativamente à adubação realizada a lanço, e o aumento da concentração de nutrientes nessas áreas é inversamente proporcional ao espaçamento entre as linhas de plantas. Neste estudo, simulou-se a aplicação dos fertilizantes em linhas, distanciadas em $0,7 \mathrm{~m}$, cujos fertilizantes seriam distribuídos numa faixa lateral de $2,5 \mathrm{~cm}$, totalizando uma área de contato dos fertilizantes com o solo de $357 \mathrm{~m}^{2}$. Em decorrência disso, as quantidades aplicadas de SFT, DAP e $\mathrm{KCl}$ em cada unidade experimental foram, respectivamente, 10,47; 9,56; e 7,33 g.

Não foi aplicado corretivo de acidez do solo em nenhum tratamento, uma vez que o objetivo era avaliar o comportamento dos nutrientes em solos bastante ácidos. A concentração de alguns nutrientes no SFT, DAP e KCl, respectivamente, era: $\mathrm{P}=183$, 200 e $0 \mathrm{~g} \mathrm{~kg}^{-1} ; \mathrm{K}=3,2$ e $480 \mathrm{~g} \mathrm{~kg}^{-1} ; \mathrm{Ca}=146,16$ e $0,1 \mathrm{~g} \mathrm{~kg}^{-1} ; \mathrm{e} \mathrm{Mg}=10$, 15 e $0,3 \mathrm{~g} \mathrm{~kg}^{-1}$.

Os solos de cada unidade experimental foram umedecidos até a capacidade de campo, antes de serem colocados nas colunas. A movimentação de P nos solos foi avaliada durante 45 dias; nesse período, as amostras de solo foram mantidas com o mesmo teor 
Quadro 1. Características físicas e químicas dos solos utilizados. Média de três repetições

\begin{tabular}{lcc}
\hline Característica & Cambissolo Húmico & Nitossolo Vermelho \\
\hline Argila $\left(\mathrm{g} \mathrm{kg}^{-1}\right)$ & 400 & 790 \\
$\mathrm{pH}\left(\mathrm{H}_{2} \mathrm{O}\right)$ & 4,5 & 4,6 \\
Matéria orgânica $\left(\mathrm{g} \mathrm{kg}^{-1}\right)$ & 50 & 23 \\
$\mathrm{P}$ Mehlich-1 $\left(\mathrm{mg} \mathrm{dm}^{-3}\right)$ & 5,3 & 1,5 \\
$\mathrm{P}$ resina $\left(\mathrm{mg} \mathrm{dm}^{-3}\right)$ & 6,7 & 6,5 \\
$\mathrm{~K}\left(\mathrm{mg} \mathrm{dm}^{-3}\right)$ & 182 & 158 \\
$\mathrm{Al}\left(\mathrm{cmol}_{\mathrm{c}} \mathrm{dm}^{-3}\right)^{(2)}$ & 3,4 & 2,5 \\
$\mathrm{Ca}\left(\mathrm{cmol}_{\mathrm{c}} \mathrm{dm}^{-3}\right)$ & 0,6 & 0,5 \\
$\mathrm{Mg}\left(\mathrm{cmol}_{\mathrm{c}} \mathrm{dm}^{-3}\right)$ & 0,5 & 0,4 \\
$\mathrm{CTC}$ efetiva $\left(\mathrm{cmol}_{\mathrm{c}} \mathrm{dm}^{-3}\right)$ & 6,2 & 3,9 \\
$\mathrm{CTC} \mathrm{pH} 7,0\left(\mathrm{cmol}_{\mathrm{c}} \mathrm{dm}^{-3}\right)$ & 30,1 & 20,7 \\
$\mathrm{Fe} \mathrm{O}_{3}\left(\mathrm{~g} \mathrm{~kg}^{-1}\right)$ & 40 & 180 \\
$\mathrm{CMAP}(\mathrm{mg} \mathrm{dm})^{(3)}$ & 831 & 2195 \\
$\mathrm{CMEP}(\mathrm{RTA})\left(\mathrm{mg} \mathrm{dm}^{-3}\right)^{(4)}$ & 800 & 800 \\
\hline
\end{tabular}

(1) Avaliações analíticas realizadas com os métodos descritos por Tedesco et al. (1995). ${ }^{(2)}$ O Al foi determinado por titulometria usando-se fenolftaleína como indicador do ponto de viragem. ${ }^{(3)}$ CMAP: capacidade máxima de adsorção de fósforo obtida pela equação de Langmuir. ${ }^{(4)} \mathrm{CMEP}$ (RTA): capacidade de extração máxima de P obtida pelo método da resina trocadora de ânions.

de umidade, pois as colunas foram acondicionadas dentro de sacos plásticos vedados, que foram abertos semanalmente para oxigenação e pesagens. Ao final do período experimental, cada coluna foi separada transversalmente, na intersecção dos anéis, e o volume de cada anel passou a constituir uma amostra de solo da respectiva distância, a partir do local de aplicação dos fertilizantes.

Em cada uma das distâncias compreendidas pelos anéis (até a camada de $5-10 \mathrm{~cm}$ ), foi quantificado o Pdisponível, extraído por solução ácida diluída (Mehlich-1) e por resina trocadora de ânions (RTA), cuja determinação foi feita pelo método de Murphy \& Riley (1962). Após observar que o P se movimentou até o quarto centímetro distante do local de aplicação dos fertilizantes, realizaram-se nove extrações sucessivas, uma por dia, com cada método, nas amostras distantes de $0,0-1,0 ; 1,0-2,0 ; 2,0-3,0 ;$ e 3,0 $4,0 \mathrm{~cm}$ dos locais de aplicação dos fertilizantes. Avaliaram-se ainda os valores de $\mathrm{pH}\left(\mathrm{H}_{2} \mathrm{O}\right)$ e de $\mathrm{Al}, \mathrm{K}$, Ca e Mg trocáveis, de acordo com Tedesco et al. (1995). $\mathrm{O}$ valor de $\mathrm{Al}$ foi determinado por titulação, com $\mathrm{NaOH}$; Ca e Mg, por espectrofotometria de absorção atômica; e K, por fotometria de emissão.

Utilizou-se o delineamento inteiramente casualizado, com quatro repetições. Os resultados de $\mathrm{pH}, \mathrm{Al}, \mathrm{K}, \mathrm{Ca}$ e Mg foram avaliados estatisticamente por meio de análise da variância $(p<0,05)$. Quando houve significância, as médias foram comparadas pelo teste de Tukey a $5 \%$. As curvas de dessorção acumulada de $\mathrm{P}$, foram ajustadas pela equação $y=\beta-(\beta-\alpha) e^{-k t}$, proposta por McKean \& Warren (1996). Nela, $y$ é o teor de $\mathrm{P}$ dessorvido; $\beta$, a dessorção máxima; $\alpha$, a dessorção na primeira extração; $k$, a taxa de dessorção constante; e $t$, o tempo de extração.
Os valores totais de $\mathrm{P}$ dessorvidos em cada uma das camadas avaliadas foram submetidos à análise de variância e ao teste de comparação de médias (Tukey, $\mathrm{p}<0,05)$. Os dados estatísticos referentes às comparações de $\mathrm{P}$ dessorvido entre fertilizantes nas camadas não são apresentados.

\section{RESULTADOS E DISCUSSÃO}

\section{Efeito no pH}

$\mathrm{O} \mathrm{KCl}$ diminui o $\mathrm{pH}\left(\mathrm{H}_{2} \mathrm{O}\right)$ do solo nas proximidades das regiões fertilizadas (Quadro 2). Apesar de não haver nenhum tratamento sem fertilizantes, naquele que recebeu apenas $\mathrm{KCl}$, aqui considerado como controle, o pH diminui uniformemente em todas as distâncias avaliadas, até $10 \mathrm{~cm}$ da localização dos grânulos, nos dois solos (Quadros 1 e 2). No CH, o valor original do $\mathrm{pH}$ era 4,5 e diminuiu para 3,6 , enquanto no NV ele diminuiu de 4,6 para 3,9. Essa diminuição de $\mathrm{pH}$ está principalmente relacionada com o aumento da densidade de cargas elétricas que ocorre nas superfícies sólidas de solos com predomínio de carga líquida negativa, em decorrência do aumento da concentração eletrolítica promovida pelo $\mathrm{KCl}$ (Neves et al., 2009). A hidrólise do Al, após seu deslocamento das cargas negativas pelo $\mathrm{K}$ aplicado, também contribui para a diminuição do $\mathrm{pH}$ (Ernani et al., 2004). O teor de K nas amostras de solo previamente à aplicação dos tratamentos era de $189 \mathrm{mg} \mathrm{dm}^{-3}$ no $\mathrm{CH}$ e $158 \mathrm{mg} \mathrm{dm}^{-3}$ no NV, o qual aumentou, respectivamente, para até 3.475 e $3.920 \mathrm{mg} \mathrm{dm}^{-3}$, na camada mais distante $(5-10 \mathrm{~cm})$ do local de aplicação do KCl (Quadros 1 e 3). Neves et al. (2009) simularam 
Quadro 2. Valores de $\mathrm{pH}\left(\mathrm{H}_{2} \mathrm{O}\right)$ e alumínio trocável ( $\left.\mathrm{Al}^{3+}\right)$ nos solos em diferentes distâncias a partir do local de aplicação dos fertilizantes. Média de quatro repetições

\begin{tabular}{|c|c|c|c|c|c|c|c|}
\hline \multirow[t]{2}{*}{ Tratamento $^{(1)}$} & \multicolumn{7}{|c|}{ Camada distante do fertilizante $(\mathrm{cm})$} \\
\hline & $0-1$ & $1-2$ & $2-3$ & $3-4$ & 4-5 & $5-10$ & $\mathbf{C V}^{(2)}$ \\
\hline & & & & & & & $\%$ \\
\hline & \multicolumn{7}{|c|}{$\mathrm{pH}\left(\mathrm{H}_{2} \mathrm{O}\right)$ - Cambissolo } \\
\hline $\mathrm{SFT}+\mathrm{KCl}$ & $4,4 \mathrm{Bb}$ & $5,1 \mathrm{Ba}$ & $4,4 \mathrm{Bb}$ & $3,8 \mathrm{c}$ & $3,6 \mathrm{Ac}$ & $3,7 \mathrm{Ac}$ & 3,8 \\
\hline $\mathrm{DAP}+\mathrm{KCl}$ & $7,1 \mathrm{Aa}$ & $6,9 \mathrm{Aa}$ & $5,8 \mathrm{Ab}$ & $3,8 \mathrm{c}$ & $3,7 \mathrm{Ac}$ & $3,6 \mathrm{ABc}$ & 3,5 \\
\hline $\mathrm{KCl}$ & $3,6 \mathrm{C}$ & $3,6 \mathrm{C}$ & $3,6 \mathrm{C}$ & 3,6 & $3,5 \mathrm{~B}$ & $3,5 \mathrm{~B}$ & 1,8 \\
\hline \multirow[t]{2}{*}{$\mathrm{CV}(\%)^{(3)}$} & 4,1 & 2,5 & 4,1 & 2,4 & 2,0 & 2,9 & \\
\hline & \multicolumn{7}{|c|}{$\mathrm{pH}\left(\mathrm{H}_{2} \mathrm{O}\right)$ - Nitossolo } \\
\hline $\mathrm{SFT}+\mathrm{KCl}$ & $5,1 \mathrm{Bb}$ & $5,6 \mathrm{Ba}$ & $5,0 \mathrm{Ab}$ & $4,0 \mathrm{Bc}$ & $3,9 \mathrm{ABc}$ & $3,9 \mathrm{c}$ & 4,7 \\
\hline $\mathrm{DAP}+\mathrm{KCl}$ & $7,2 \mathrm{Aa}$ & 7,0 Aab & $6,0 \mathrm{Ab}$ & $4,8 \mathrm{Ac}$ & $4,0 \mathrm{Ac}$ & $3,9 \mathrm{c}$ & 8,4 \\
\hline $\mathrm{KCl}$ & $3,9 \mathrm{C}$ & $3,9 \mathrm{C}$ & $3,8 \mathrm{~B}$ & $3,8 \mathrm{~B}$ & $3,8 \mathrm{~B}$ & 3,9 & 2,1 \\
\hline \multirow[t]{2}{*}{$\mathrm{CV}(\%)^{(3)}$} & 1,9 & 4,6 & 14,3 & 11,1 & 3,4 & 2,8 & \\
\hline & \multicolumn{7}{|c|}{$\mathrm{Al}^{3+}\left(\mathrm{cmol}_{\mathrm{c}} \mathrm{dm}^{-3}\right)^{(4)}-$ Cambissolo } \\
\hline $\mathrm{SFT}+\mathrm{KCl}$ & $2,9 \mathrm{Bbc}$ & $0,8 \mathrm{Bd}$ & $0,58 \mathrm{Bd}$ & $2,4 \mathrm{Bc}$ & $3,8 \mathrm{Bab}$ & $4,8 \mathrm{a}$ & 18,4 \\
\hline $\mathrm{DAP}+\mathrm{KCl}$ & $\mathrm{ND}^{(5)}$ & ND & ND & $3,0 \mathrm{Bbc}$ & $3,8 \mathrm{Bb}$ & $5,2 \mathrm{a}$ & 17,7 \\
\hline $\mathrm{KCl}$ & $4,4 \mathrm{Ab}$ & 4,6 Aab & 4,7 Aab & $5,4 \mathrm{Aa}$ & $5,4 \mathrm{Aa}$ & $5,4 \mathrm{a}$ & 8,2 \\
\hline \multirow[t]{2}{*}{$\mathrm{CV}(\%)^{(3)}$} & 14,0 & 25,1 & 23,4 & 16,4 & 16,2 & 11,0 & \\
\hline & \multicolumn{7}{|c|}{$\mathrm{Al}^{3+}\left(\mathrm{cmol}_{\mathrm{c}} \mathrm{dm}^{-3}\right)-$ Nitossolo } \\
\hline $\mathrm{SFT}+\mathrm{KCl}$ & $1,0 \mathrm{Ba}$ & $0,4 \mathrm{Bc}$ & $0,2 \mathrm{Bc}$ & 0,6 Bbc & $0,8 \mathrm{Bab}$ & $1,0 \mathrm{a}$ & 30,6 \\
\hline $\mathrm{DAP}+\mathrm{KCl}$ & ND & ND & ND & $1,0 \mathrm{~A}$ & $1,2 \mathrm{~A}$ & 1,2 & 31,3 \\
\hline $\mathrm{KCl}$ & $1,1 \mathrm{AB}$ & $1,0 \mathrm{AB}$ & $1,2 \mathrm{~A}$ & $1,0 \mathrm{~A}$ & $1,0 \mathrm{AB}$ & 1,4 & 34,4 \\
\hline $\mathrm{CV}(\%)^{(3)}$ & 39,7 & 49,1 & 37,7 & 25,0 & 14,4 & 22,9 & \\
\hline
\end{tabular}

(1) SFT: superfosfato triplo; DAP: diamônio fosfato; KCl: cloreto de potássio; ${ }^{(2)} \mathrm{CV}$ : coeficiente de variação do fator camada; ${ }^{(3)} \mathrm{CV}$ : coeficiente de variação do fator fonte; ${ }^{(4)} \mathrm{O} \mathrm{Al}$ foi extraído com solução de $\mathrm{KCl} 1,0 \mathrm{~mol} \mathrm{~L}^{-1}$ e determinado utilizando azul de bromotimol como indicador do ponto de viragem; e ${ }^{(5)} \mathrm{ND}$ : não detectável. Médias seguidas de letras maiúsculas distintas, nas colunas, e minúsculas, nas linhas, diferem entre si pelo teste de Tukey a $5 \%$. Ausência de letra representa inexistência de diferença estatística.

a aplicação localizada de $\mathrm{KCl}$ em cinco solos com diferentes tamponamentos e verificaram que o K migrou para distâncias maiores do que $4,0 \mathrm{~cm}$ dos locais de aplicação em apenas sete dias.

$\mathrm{O}$ efeito dos fosfatos no $\mathrm{pH}$ do solo variou com o fertilizante, com o tipo de solo e com a distância dos grânulos (Quadro 2). Quando adicionados juntamente com KCl, os dois fosfatos (SFT e DAP) aumentaram o $\mathrm{pH}$ até a camada de 2,0-3,0 cm distante dos grânulos, nos dois solos, relativamente ao tratamento só com $\mathrm{KCl}$ (Quadro 2). Na camada de 0,0-1,0 cm, em relação ao tratamento com $\mathrm{KCl}$, o $\mathrm{pH}$ do Cambissolo aumentou de 3,6 para até 4,4, onde foi aplicado SFT + $\mathrm{KCl}$, e para até 7,1 , no tratamento que recebeu DAP + KCl. No Nitossolo, o pH aumentou de 3,9, para até 5,1 , onde foi aplicado SFT $+\mathrm{KCl}$, e para até 7,2 , no tratamento que recebeu DAP + KCl. Diferentemente do que ocorreu no tratamento só com $\mathrm{KCl}$, onde não houve diferença de $\mathrm{pH}$ entre as camadas, o $\mathrm{pH}$ dos solos não foi influenciado pela adição dos fertilizantes fosfatados nas camadas de solo com distância superior a $3 \mathrm{~cm}$ dos grânulos (Quadro 2), evidenciando que os produtos resultantes da dissolução dos fosfatos tiveram baixa mobilidade.

A elevação do $\mathrm{pH}$ do solo ocasionada pelo DAP ocorre em razão do consumo de hidrogênio $\left(\mathrm{H}^{+}\right)$decorrente da transformação de parte das moléculas de $\mathrm{HPO}_{4}{ }^{2-}$ desse adubo em moléculas de $\mathrm{H}_{2} \mathrm{PO}_{4}^{-}$(Ernani et al., 2002; Ernani, 2008). Essa reação acontece por causa do equilíbrio químico existente entre essas duas formas fosfatadas $\left(\mathrm{H}_{2} \mathrm{PO}_{4}^{-}=\mathrm{HPO}_{4}{ }^{2-}+\mathrm{H}^{+}\right)$, cuja constante de dissociação ácida é $6,2 \times 10^{-8}$, o que resulta num $\mathrm{pK}_{\mathrm{a}}$ (potencial da constante de dissociação) de 7,21. Sendo assim, quando o $\mathrm{pH}$ do solo for 7,21 o meio terá $50 \%$ de cada forma; abaixo e acima dele, haverá predomínio de $\mathrm{H}_{2} \mathrm{PO}_{4}{ }^{-}$e $\mathrm{HPO}_{4}{ }^{2-}$, respectivamente (Ernani, 2008). A aplicação de SFT, entretanto, normalmente diminui o pH do solo ao redor dos grânulos desse adubo (Ernani et al., 2002; Mantovani et al., 2007) em razão da liberação de íons $\mathrm{H}^{+}$por ocasião da dissolução de suas moléculas $\left[\left(\mathrm{H}_{2} \mathrm{PO}_{4}\right)_{2}=\mathrm{H}_{2} \mathrm{PO}_{4}+\mathrm{HPO}_{4}^{-}+\mathrm{H}^{+}\right]$. A elevação do $\mathrm{pH}$ do solo ocasionada por esse fertilizante neste estudo (Quadro 2) provavelmente ocorreu porque as reações de adsorção específica das moléculas de 
$\mathrm{H}_{2} \mathrm{PO}_{4}^{-}$e $\mathrm{HPO}_{4}{ }^{2-}$ liberaram maior quantidade de $\mathrm{OH}^{-}$ do que o número de átomos de $\mathrm{H}^{+}$liberados pela dissociação do $\mathrm{H}_{2} \mathrm{PO}_{4}^{-}$, uma vez que apenas parte dessas moléculas se transforma em $\mathrm{HPO}_{4}{ }^{-}$.

$\mathrm{O}$ efeito dos fosfatos no comportamento do $\mathrm{pH}$ com a variação da distância a partir da região fertilizada diferiu entre esses. O DAP elevou o $\mathrm{pH}$ do solo com o aumento da proximidade dos grânulos, porém, onde foi aplicado SFT, o pH na camada com os grânulos (0$1,0 \mathrm{~cm}$ ) foi menor do que na camada imediatamente seguinte $(1,0-2,0 \mathrm{~cm})$.

\section{Efeito no Al}

O efeito da adição de altas doses de $\mathrm{KCl}$ nos teores de alumínio trocável $\left(\mathrm{Al}^{3+}\right)$ variou entre os solos (Quadro 2). $\mathrm{No} \mathrm{NV}, \mathrm{o} \mathrm{Al}{ }^{3+}$ não foi influenciado pela adição de $\mathrm{KCl}$ em nenhuma das distâncias, a partir do local de aplicação com média de $1,2 \mathrm{cmol}_{\mathrm{c}} \mathrm{dm}^{-3}$. No $\mathrm{CH}$, no entanto, a concentração de $\mathrm{Al}^{3+}$ diminuiu com a aproximação do local de aplicação dos grânulos, o qual variou de 5,4 $\mathrm{cmol}_{\mathrm{c}} \mathrm{dm}^{-3}$, nas camadas de $3,0-4,0 ; 4,0-5,0$ e $5,0-10,0 \mathrm{~cm}$ distante da região fertilizada, para $4,4 \mathrm{cmol}_{\mathrm{c}} \mathrm{dm}^{-3}$, na camada com o fertilizante $(0,0-1,0 \mathrm{~cm})$.

Os dois fertilizantes fosfatados diminuíram os teores de $\mathrm{Al}^{3+}$ nas adjacências dos grânulos no Cambissolo, porém só o $\mathrm{DAP}$ diminuiu o $\mathrm{Al}$ no Nitossolo (Quadro 2). Nos dois solos, o DAP eliminou o $\mathrm{Al}$ até a camada distante $3,0 \mathrm{~cm}$ dos grânulos e isso ocorreu principalmente em razão da elevação do $\mathrm{pH}$ proporcionado por esse fertilizante nessas camadas (Quadro 2). O SFT, no entanto, somente diminuiu o Al no Cambissolo também até $3,0 \mathrm{~cm}$ distante dos grânulos, porém o menor decréscimo foi verificado junto a esses (camada de 0,0-1,0 cm).

\section{Efeito no Ca}

A adição de altas doses de $\mathrm{KCl}$ teve pequeno efeito nos teores de Ca nas diferentes distâncias, a partir do local de aplicação do fertilizante. Na comparação das distâncias, o Ca variou de 0,3 a $0,6 \mathrm{cmol}_{\mathrm{c}} \mathrm{dm}^{-3}$ no $\mathrm{CH}$ e de 0,4 a $0,6 \mathrm{cmol}_{\mathrm{c}} \mathrm{dm}^{-3}$ no NV (Quadro 3). A aplicação de DAP, juntamente com $\mathrm{KCl}$, também não interferiu nos valores de $\mathrm{Ca}$ em nenhuma distância avaliada relativamente ao tratamento com $\mathrm{KCl}$, nos dois solos (Quadro 3). O SFT, entretanto, por apresentar Ca em sua composição, elevou as concentrações de $\mathrm{Ca}$ em todas as distâncias avaliadas, nos dois solos, cujos valores foram decrescendo com o aumento da distância a partir do local de aplicação do adubo (Quadro 3). No $\mathrm{CH}$, o $\mathrm{Ca}^{2+}$ na camada mais próxima do SFT (0,0-1,0 cm) atingiu $13,9 \mathrm{cmol}_{\mathrm{c}} \mathrm{dm}^{-3}$, tendo diminuído para $2,6 \mathrm{cmol}_{\mathrm{c}} \mathrm{dm}^{-3}$ na camada mais distante (5,0-10,0 cm); no NV, o $\mathrm{Ca}^{2+}$ nessas mesmas camadas foi 6,0 e $3,5 \mathrm{cmol}_{\mathrm{c}} \mathrm{dm}^{-3}$, respectivamente. Nos dois solos, entretanto, o teor de Ca na camada mais distante dos grânulos de SFT $(5-10 \mathrm{~cm})$ foi maior do que no solo só com $\mathrm{KCl}$, evidenciando que o $\mathrm{Ca}$ aplicado por esse fosfato se movimentou até mais de $10 \mathrm{~cm}$ do local de aplicação. A quantidade de Ca adicionada pelo SFT foi a mesma nos dois solos; entretanto, até a camada de 2,0-3,0 cm distante dos grânulos, o teor de $\mathrm{Ca}^{2+}$ foi praticamente duas vezes maior no $\mathrm{CH}$ do que no NV, possivelmente decorrente da maior CTC do $\mathrm{CH}$, relativamente ao NV, que assim permitiu menor mobilidade desse cátion.

\section{Efeito do Mg}

A adição de altas doses do fertilizante potássico (KCl) não alterou a concentração do $\mathrm{Mg}^{2+}$ em nenhuma distância a partir do local de aplicação do adubo, independentemente do solo (Quadro 3). Os valores de $\mathrm{Mg}^{2+}$, na média de todas as distâncias, foram $0,3 \mathrm{e}$ $0,4 \mathrm{cmol}_{\mathrm{c}} \mathrm{dm}^{-3}$, respectivamente no $\mathrm{CH}$ e NV.

$\mathrm{O}$ efeito dos fertilizantes fosfatados nos teores de $\mathrm{Mg}^{2+}$ variou com a fonte de P (Quadro 3). O SFT aumentou o $\mathrm{Mg}^{2+}$ em todas as camadas avaliadas em relação ao tratamento com somente $\mathrm{KCl}$, nos dois solos: no $\mathrm{CH}$, o $\mathrm{Mg}$ passou de 0,3 para $1,5 \mathrm{cmol}_{\mathrm{c}} \mathrm{dm}^{-3}$ e no $\mathrm{NV}$ de 0,4 para $1,2 \mathrm{cmol}_{\mathrm{c}} \mathrm{dm}^{-3}$. Isso ocorreu em razão da presença de $1,0 \% \mathrm{Mg}$ nesse fertilizante. A adição de DAP, juntamente com $\mathrm{KCl}$, no entanto, não influenciou o $\mathrm{Mg}$ do solo em relação ao tratamento só com KCl (Quadro 3). Na comparação entre camadas, entretanto, nas proximidades dos grânulos de DAP, o $\mathrm{Mg}^{2+}$ foi menor do que nas camadas mais distantes, nos dois solos (Quadro 3), provavelmente por causa da formação de precipitados com $\mathrm{P}$, reação essa favorecida em valores altos de $\mathrm{pH}$, que foram superiores a 7,0 nas duas primerias camadas onde foi adicionado DAP ao solo (Quadro 2).

\section{Efeito no K}

O K teve boa mobilidade nos dois solos, pois a aplicação de $\mathrm{KCl}$, isolada ou associada com os fosfatos, aumentou a concentração de K em todas as distâncias avaliadas (Quadro 3). A adição localizada de uma dose equivalente a $200 \mathrm{~kg} \mathrm{ha}^{-1}$ de $\mathrm{K}_{2} \mathrm{O}(3,6 \mathrm{~g}$ de $\mathrm{K})$ proporcionou valores de $\mathrm{K}$ na camada de solo mais próxima dos grânulos $(0,0-1,0 \mathrm{~cm})$, que variaram de 9.754 a $10.125 \mathrm{mg} \mathrm{dm}^{-3}$, respectivamente no $\mathrm{CH}$ e $\mathrm{NV}$; na camada mais distante do local fertilizado $(5,0-$ $10,0 \mathrm{~cm}$ ), as concentrações de K nesses solos foram, respectivamente de 3.475 e $3.920 \mathrm{mg} \mathrm{dm}^{-3}$ (Quadro 3).

Nas proximidades dos fertilizantes, até $2,0 \mathrm{~cm}$ distante dos grânulos, os teores de $\mathrm{K}$ não foram influenciados pela adição dos adubos fosfatados, em nenhum solo. Nas demais regiões, entretanto, os tratamentos que receberam fosfatos proporcionaram menor mobilidade do $\mathrm{K}$, uma vez que os teores foram menores do que onde foi aplicado somente $\mathrm{KCl}$ (Quadro 3). Esse decréscimo na mobilidade de K pode estar associado à formação de alguns precipitados de fosfatos de potássio (Akinremi \& Cho, 1993).

\section{Efeito no P}

A quantidade de $\mathrm{P}$ na camada mais próxima do local de aplicação dos fertilizantes, diagnosticada por uma única extração com o método Mehlich-1, divergiu 
Quadro 3. Teores de Ca, Mg e K nos solos em diferentes distâncias a partir do local de aplicação dos fertilizantes. Média de quatro repetições

\begin{tabular}{|c|c|c|c|c|c|c|c|}
\hline \multirow[t]{2}{*}{ Tratamento $^{(1)}$} & \multicolumn{7}{|c|}{ Camada distante do fertilizante $(\mathrm{cm})$} \\
\hline & $0-1$ & $1-2$ & $2-3$ & $3-4$ & $4-5$ & $5-10$ & $\mathbf{C V}^{(2)}$ \\
\hline & & & & & & & $\%$ \\
\hline & \multicolumn{7}{|c|}{$\mathrm{Ca}\left(\mathrm{cmol}_{\mathrm{c}} \mathrm{dm}^{-3}\right)-$ Cambissolo } \\
\hline $\mathrm{SFT}+\mathrm{KCl}$ & $13,9 \mathrm{Aa}$ & $11,5 \mathrm{Ab}$ & $8,2 \mathrm{Ac}$ & $5,6 \mathrm{Ad}$ & $3,6 \mathrm{Ae}$ & $2,6 \mathrm{Ae}$ & 9,2 \\
\hline $\mathrm{DAP}+\mathrm{KCl}$ & $0,8 \mathrm{Bb}$ & 1,0 Bab & 1,2 Bab & 1,2 Bab & $1,4 \mathrm{Ba}$ & $1,4 \mathrm{Ba}$ & 23,6 \\
\hline $\mathrm{KCl}$ & $0,3 \mathrm{Bc}$ & $0,3 \mathrm{Bc}$ & $0,4 \mathrm{Bbc}$ & $0,5 \mathrm{Ba}$ & $0,5 \mathrm{Ba}$ & $0,6 \mathrm{Ba}$ & 14,2 \\
\hline \multirow[t]{2}{*}{$\mathrm{CV}^{(3)}$} & 13,2 & 18,9 & 16,3 & 20,4 & 21,9 & 23,6 & \\
\hline & \multicolumn{7}{|c|}{$\mathrm{Ca}\left(\mathrm{cmol}_{\mathrm{c}} \mathrm{dm}^{-3}\right)-$ Nitossolo } \\
\hline $\mathrm{SFT}+\mathrm{KCl}$ & $6,0 \mathrm{Aa}$ & 5,9 Aab & 4,6 Aabc & 4,5 Aabc & $4,4 \mathrm{Abc}$ & $3,5 \mathrm{Ac}$ & 13,8 \\
\hline $\mathrm{DAP}+\mathrm{KCl}$ & $0,8 \mathrm{~B}$ & $0,9 \mathrm{~B}$ & $1,0 \mathrm{~B}$ & $1,2 \mathrm{~B}$ & $1,3 \mathrm{~B}$ & $1,3 \mathrm{~B}$ & 34,7 \\
\hline $\mathrm{KCl}$ & $0,6 \mathrm{Ba}$ & $0,6 \mathrm{Ba}$ & $0,4 \mathrm{Bb}$ & $0,4 \mathrm{Bb}$ & $0,4 \mathrm{Bb}$ & $0,5 \mathrm{Bab}$ & 20,2 \\
\hline \multirow[t]{2}{*}{$\mathrm{CV}^{(3)}$} & 13,9 & 16,2 & 35,3 & 29,9 & 32,3 & 19,5 & \\
\hline & \multicolumn{7}{|c|}{$\mathrm{Mg}\left(\mathrm{cmol}_{\mathrm{c}} \mathrm{dm}^{-3}\right)$ - Cambissolo } \\
\hline $\mathrm{SFT}+\mathrm{KCl}$ & $1,6 \mathrm{~A}$ & $1,5 \mathrm{~A}$ & $1,6 \mathrm{~A}$ & $1,4 \mathrm{~A}$ & $1,4 \mathrm{~A}$ & $1,4 \mathrm{~A}$ & 9,8 \\
\hline $\mathrm{DAP}+\mathrm{KCl}$ & $0,1 \mathrm{Be}$ & 0,3 Bde & $0,6 \mathrm{Bab}$ & $0,5 \mathrm{Bbc}$ & $0,8 \mathrm{Ba}$ & $0,4 \mathrm{Bcd}$ & 20,6 \\
\hline $\mathrm{KCl}$ & $0,3 \mathrm{~B}$ & $0,3 \mathrm{~B}$ & $0,3 \mathrm{~B}$ & $0,4 \mathrm{~B}$ & $0,4 \mathrm{C}$ & $0,3 \mathrm{~B}$ & 21,0 \\
\hline \multirow[t]{2}{*}{$\mathrm{CV}^{(3)}$} & 8,1 & 6,5 & 12,0 & 9,5 & 22,1 & 13,9 & \\
\hline & \multicolumn{7}{|c|}{$\mathrm{Mg}\left(\mathrm{cmol}_{\mathrm{c}} \mathrm{dm}^{-3}\right)-$ Nitossolo } \\
\hline $\mathrm{SFT}+\mathrm{KCl}$ & $1,5 \mathrm{Aa}$ & $1,4 \mathrm{Aa}$ & $1,3 \mathrm{Ab}$ & $1,1 \mathrm{Ac}$ & $1,1 \mathrm{Ac}$ & $0,9 \mathrm{Ad}$ & 4,6 \\
\hline $\mathrm{DAP}+\mathrm{KCl}$ & $0,2 \mathrm{Cc}$ & $0,3 \mathrm{Bbc}$ & $0,3 \mathrm{Bbc}$ & $0,4 \mathrm{Bab}$ & $0,4 \mathrm{Bab}$ & $0,5 \mathrm{Ba}$ & 21,7 \\
\hline $\mathrm{KCl}$ & $0,4 \mathrm{~B}$ & $0,4 \mathrm{~B}$ & $0,4 \mathrm{~B}$ & $0,3 \mathrm{C}$ & $0,4 \mathrm{~B}$ & $0,3 \mathrm{C}$ & 20,4 \\
\hline \multirow[t]{2}{*}{$\mathrm{CV}^{(3)}$} & 11,4 & 10,1 & 15,0 & 6,8 & 4,5 & 20,6 & \\
\hline & \multicolumn{7}{|c|}{$\mathrm{K}\left(\mathrm{mg} \mathrm{dm}^{-3}\right)-$ Cambissolo } \\
\hline $\mathrm{SFT}+\mathrm{KCl}$ & $10850 \mathrm{a}$ & $8725 \mathrm{~b}$ & $6700 \mathrm{ABc}$ & $5275 \mathrm{Ac}$ & $3775 \mathrm{Bd}$ & $2085 \mathrm{Be}$ & 10,4 \\
\hline $\mathrm{DAP}+\mathrm{KCl}$ & $11125 \mathrm{a}$ & $9750 \mathrm{~b}$ & $6400 \mathrm{Bc}$ & $3875 \mathrm{Bd}$ & $2675 \mathrm{Ce}$ & $1565 \mathrm{Ce}$ & 9,9 \\
\hline $\mathrm{KCl}$ & $9754 \mathrm{a}$ & $8800 \mathrm{ab}$ & $7575 \mathrm{Ab}$ & $6300 \mathrm{Ac}$ & $5500 \mathrm{Ac}$ & $3475 \mathrm{Ad}$ & 6,8 \\
\hline \multirow[t]{2}{*}{$\mathrm{CV}^{(3)}$} & 6,9 & 9,4 & 8,3 & 13,2 & 9,0 & 11,9 & \\
\hline & \multicolumn{7}{|c|}{$\mathrm{K}\left(\mathrm{mg} \mathrm{dm} \mathrm{m}^{-3}\right)-$ Nitossolo } \\
\hline $\mathrm{SFT}+\mathrm{KCl}$ & $10175 \mathrm{a}$ & $8125 \mathrm{~b}$ & $6450 \mathrm{Bc}$ & $5500 \mathrm{ABc}$ & $4425 \mathrm{Bd}$ & $2218 \mathrm{Be}$ & 6,9 \\
\hline $\mathrm{DAP}+\mathrm{KCl}$ & $11175 \mathrm{a}$ & $8850 \mathrm{ab}$ & $6600 \mathrm{Bbc}$ & $4500 \mathrm{Bcd}$ & 2925 Cde & $1702 \mathrm{Be}$ & 17,6 \\
\hline $\mathrm{KCl}$ & $10125 \mathrm{a}$ & 8675 b & $7300 \mathrm{Ac}$ & 6550 Acd & $5775 \mathrm{Ad}$ & $3920 \mathrm{Ae}$ & 8,3 \\
\hline $\mathrm{CV}^{(3)}$ & 6,9 & 12,0 & 16,1 & 14,1 & 12,9 & 26,6 & \\
\hline
\end{tabular}

(1) SFT: superfosfato triplo; DAP: diamônio fosfato; $\mathrm{KCl}$ : cloreto de potássio; ${ }^{(2)} \mathrm{CV}$ : coeficiente de variação do fator camada; ${ }^{(3)} \mathrm{CV}$ : coeficiente de variação do fator fonte. Médias seguidas de letras maiúsculas distintas nas colunas e minúsculas nas linhas diferem significativamente entre si pelo teste de Tukey a $5 \%$. Ausência de letra representa a inexistência de diferença estatística.

entre os dois fosfatos, nos dois solos (Quadro 4). A aplicação de SFT proporcionou maiores valores de $\mathrm{P}$ do que a de DAP, numa magnitude de $27 \%$ no NV e de $39 \%$, no $\mathrm{CH}$. A amplitude de variação do P extraído dessa camada, na média dos dois fosfatos, foi $95 \%$ maior no $\mathrm{CH}$ do que no NV por causa do menor teor de argila, de óxidos e oxihidróxidos no $\mathrm{CH}$, relativamente ao $\mathrm{NV}$, cujos valores máximos atingiram 4.258 e $2.094 \mathrm{mg} \mathrm{kg}^{-1}$, respectivamente. As concentrações de $\mathrm{P}$ extraídas pelo método da RTA também apresentaram diferenças entre as fontes fosfatadas quando quantificadas em uma única extração na camada $0,0-1,0 \mathrm{~cm}$ (Quadro 4). No entanto, para esse método, a aplicação de DAP promoveu maiores valores de P comparado ao SFT ( $23 \%$ no NV e $59 \%$ no $\mathrm{CH})$. Para esse método, a amplitude de variação de $\mathrm{P}$ nessa camada foi $40 \%$ maior no $\mathrm{CH}$ do que no NV, na média das duas fontes fosfatadas. A aplicação somente de $\mathrm{KCl}$ não influenciou o $\mathrm{P}$ nessa camada de solo por qualquer dos métodos de extração (Quadros 1 e 4).

Em razão da alta concentração de $\mathrm{P}$ nas proximidades dos grânulos, foram realizadas nove extrações sucessivas de $\mathrm{P}$ das amostras próximas aos grânulos, uma a cada dia. Os resultados da extração cumulativa de $\mathrm{P}$ variaram com o tipo de adubo fosfatado, a camada avaliada, o tipo de solo e com o método de extração. Na camada de $0,0-1,0 \mathrm{~cm}$ distante do local de aplicação dos fertilizantes, a adição de SFT proporcionou maiores valores acumulados de $\mathrm{P}$ do que a aplicação de DAP, quando quantificados pelo Mehlich-1, nos dois solos (Figuras 1 e 2). No NV, os 
Quadro 4. Fósforo na camada de 0-1 cm distante do local de aplicação dos fertilizantes e quantificado por uma única extração com os métodos Mehlich-1 e de resina trocadora de ânions (RTA). Média de quatro repetições

\begin{tabular}{lcccc}
\hline \multirow{2}{*}{ Tratamento $^{(1)}$} & \multicolumn{2}{c}{ Mehlich-1 } & & \multicolumn{2}{c}{ RTA } \\
\cline { 2 - 3 } & Nitossolo & Cambissolo & Nitossolo & Cambissolo \\
\cline { 2 - 4 } $\mathrm{SFT}+\mathrm{KCl}$ & $2094 \mathrm{~A}$ & $4258 \mathrm{~A}$ & $1581 \mathrm{~B}$ & $1905 \mathrm{~B}$ \\
$\mathrm{DAP}+\mathrm{KCl}$ & $1644 \mathrm{~B}$ & $3064 \mathrm{~B}$ & $1940 \mathrm{~A}$ & $3035 \mathrm{~A}$ \\
$\mathrm{KCl}$ & $1,1 \mathrm{C}$ & $5,5 \mathrm{C}$ & $13,5 \mathrm{C}$ & $12,1 \mathrm{C}$ \\
$\mathrm{CV}(\%)^{(2)}$ & 8,8 & 11,3 & 9,7 & 26,3 \\
\hline
\end{tabular}

(1) SFT: superfosfato triplo; DAP: diamônio fosfato; $\mathrm{KCl}$ : cloreto de potássio; ${ }^{(2)} \mathrm{CV}$ : coeficiente de variação. Médias seguidas de letras maiúsculas distintas nas colunas diferem entre si pelo teste de Tukey a $5 \%$.

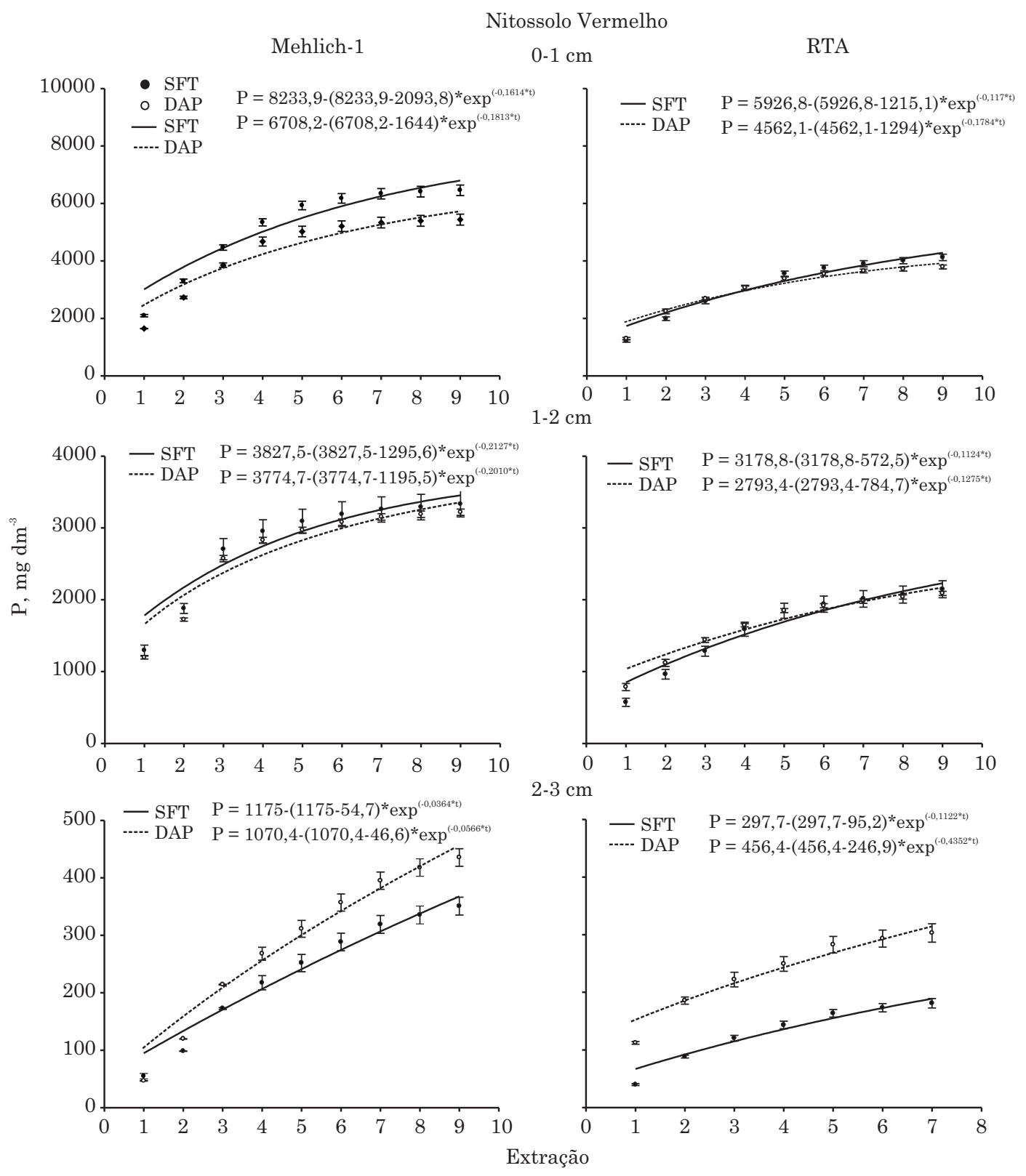

Figura 1. Dessorção acumulada de fósforo ao longo de nove extrações sucessivas em diversas camadas distante do local de aplicação dos fertilizantes em um Nitossolo Vermelho, quantificada pelos métodos Mehlich-1 e da resina trocadora de ânion (RTA). SFT: superfosfato triplo; e DAP: diamônio fosfato. 


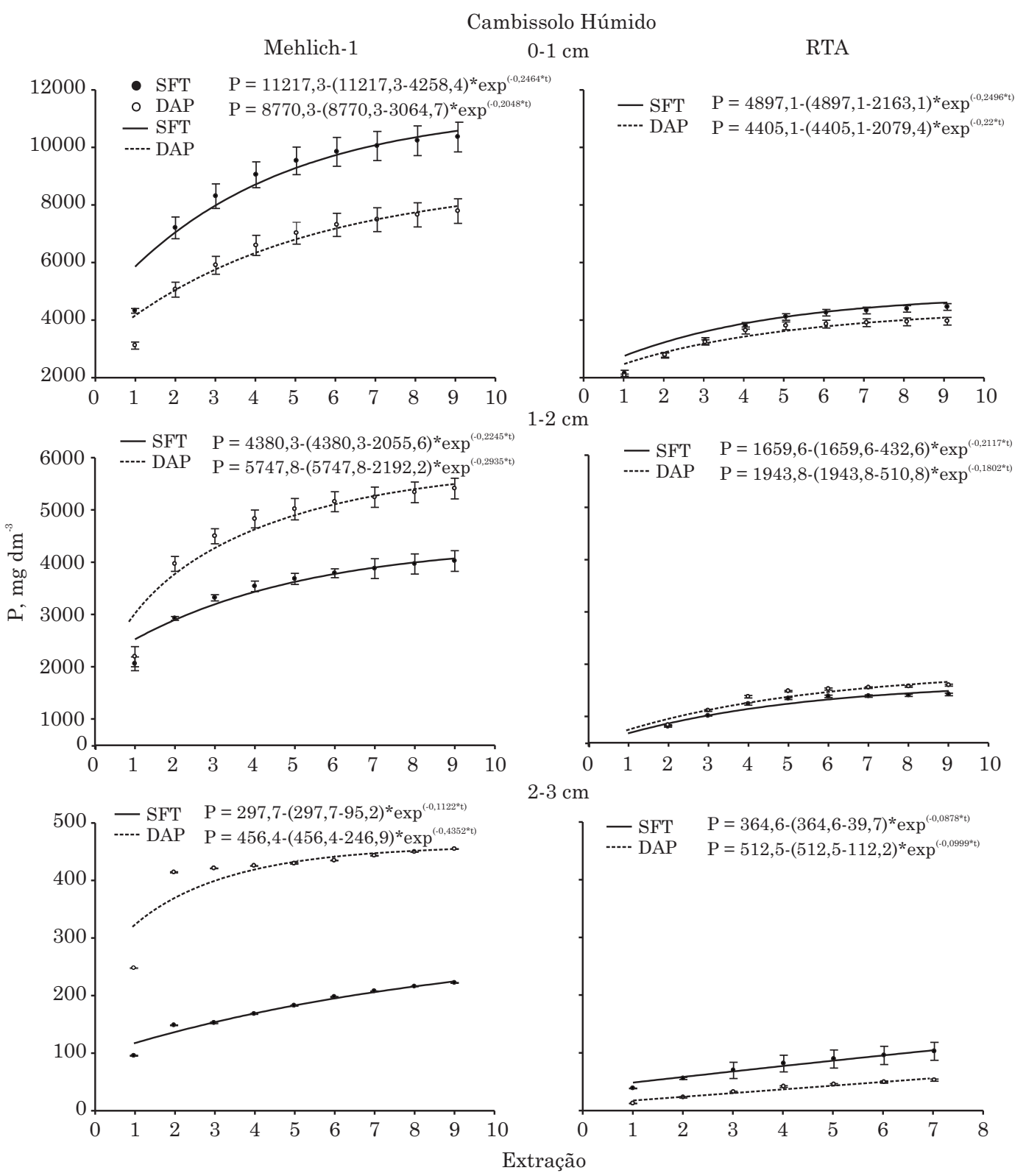

Figura 2. Dessorção acumulada de fósforo ao longo de nove extrações sucessivas em diversas camadas distante do local de aplicação dos fertilizantes em um Cambissolo Húmico, quantificada pelos métodos de Mehlich-1 e da resina trocadora de ânion (RTA). SFT: superfosfato triplo; e DAP: diamônio fosfato.

valores máximos atingidos foram de 6.518 e 5.518 $\mathrm{mg} \mathrm{dm}{ }^{-3}$, respectivamente. O P extraído pelo método da RTA, entretanto, não diferiu entre SFT e DAP em nenhuma das nove extrações, cujos valores cumulativos totalizaram 4.121 e $3.788 \mathrm{mg} \mathrm{dm}^{-3}$, respectivamente (Figura 1). No Cambissolo Húmico, o P-Mehlich-1 acumulado atingiu 10.276 e 7.650 $\mathrm{mg} \mathrm{dm}{ }^{-3}$ e o P-RTA 4.450 e $3.960 \mathrm{mg} \mathrm{dm}^{-3}$, respectivamente para SFT e DAP. Não se fez nenhuma análise adicional que esclareça a causa das diferenças entre os valores de $\mathrm{P}$ ocasionadas pelos fosfatos nas diferentes camadas. Como eles diferiram, apesar da quantidade de $\mathrm{P}$ aplicada ter sido a mesma, esse fenômeno deve ser considerado em futuros estudos de calibração de $\mathrm{P}$, visando a recomendação de adubação fosfatada.

Na camada de 1,0-2,0 cm distante dos grânulos, a quantidade de $\mathrm{P}$ extraída do NV não diferenciou entre os fosfatos em nenhum método de extração, tanto no total acumulado como nas extrações individuais (Figura 1); já no $\mathrm{CH}$, o DAP proporcionou maiores valores de $\mathrm{P}$ do que o SFT (Figura 2). Na camada seguinte, distante de 2,0-3,0 cm dos grânulos, a extração de $\mathrm{P}$ no NV foi maior para o DAP do que para o SFT, pelos dois métodos: o Mehlich-1 extraiu 351 $\mathrm{mg} \mathrm{dm}-3$ de P no tratamento com SFT e $438 \mathrm{mg} \mathrm{dm}^{-3}$ naquele com DAP, enquanto a RTA extraiu 181 e $303 \mathrm{mg} \mathrm{dm}^{-3}$ de $\mathrm{P}$, respectivamente. No $\mathrm{CH}$, somente 
o Mehlich-1 quantificou diferenças na concentração de $\mathrm{P}$ entre os fosfatos (Figura 2), tendo o DAP proporcionado maior extração de $\mathrm{P}$ do que o SFT. Na camada de 3,0-4,0 cm dos grânulos, o P cumulativo somente distinguiu entre os fosfatos pelo método da RTA, onde o solo fertilizado com SFT apresentou mais $\mathrm{P}$ do que aquele com DAP (64 e $31 \mathrm{mg} \mathrm{dm}^{-3}$, respectivamente) (dados não apresentados).

Assim como nas três primeiras camadas próximas aos grânulos, na média dos primeiros $4 \mathrm{~cm}$, o Mehlich1 sempre extraiu maior quantidade cumulativa de $\mathrm{P}$ do que a RTA, nos dois solos (Figuras 1 e 2, Quadro 5). Essa maior capacidade de extração do Mehlich-1 provavelmente deve-se ao fato desse método ser ácido e assim quantificar algumas formas de $\mathrm{P}$ precipitadas, em adição ao $\mathrm{P}$ adsorvido. Os níveis críticos de suficiência de $\mathrm{P}$ para os solos dos Estados do Rio Grande do Sul e Santa Catarina pelo método de Mehlich-1 são 6, 9, 12 e $21 \mathrm{mg} \mathrm{kg}^{-1}$ para os com valores decrescentes de argila; para o método Mehlich, adota-se um único valor, de $20 \mathrm{mg} \mathrm{kg}^{-1}$, independentemente do teor de argila (CQFSRS/SC, 2004). Os dados deste estudo evidenciam que o valor do nível crítico do método da RTA, obtido para a região sem estudos de calibração, não está adequado e deve ser modificado.

\begin{tabular}{|c|c|c|c|}
\hline \multicolumn{4}{|c|}{$\begin{array}{l}\text { Quadro 5. Fósforo total quantificado por nove } \\
\text { extrações sucessivas pelos métodos Mehlich-1 e } \\
\text { resina trocadora de ânions (RTA) na camada de } \\
0-4 \mathrm{~cm} \text { distante do local de aplicação dos } \\
\text { fertilizantes, em dois solos ácidos catarinenses. } \\
\text { Média de quatro repetições }\end{array}$} \\
\hline \multirow{2}{*}{ Tratamento $^{(1)}$} & \multicolumn{2}{|c|}{$P$ total $^{(2)}$} & \multirow[b]{2}{*}{$\mathrm{CV}^{(3)}$} \\
\hline & Nitossolo & Cambissolo & \\
\hline & \multicolumn{2}{|c|}{$\mathrm{mg} \mathrm{dm}^{-3}$} & $\%$ \\
\hline SFT + KCl Mehlich-1 & $2594 \mathrm{Aa}$ & $3712 \mathrm{Aa}$ & 48,8 \\
\hline $\mathrm{DAP}+\mathrm{KCl}$ Mehlich-1 & $2320 \mathrm{Ab}$ & $3444 \mathrm{Aa}$ & 43,5 \\
\hline $\mathrm{SFT}+\mathrm{KCl} \mathrm{RTA}$ & $1555 \mathrm{Ba}$ & $1646 \mathrm{Ba}$ & 26,9 \\
\hline $\mathrm{DAP}+\mathrm{KCl} \mathrm{RTA}$ & $1649 \mathrm{Ba}$ & $1490 \mathrm{Ba}$ & 22,0 \\
\hline $\mathrm{CV}(\%)^{(4)}$ & 58,8 & 29,8 & \\
\hline
\end{tabular}

(1) SFT: superfosfato triplo; DAP: diamônio fosfato; $\mathrm{KCl}$ : cloreto de potássio; ${ }^{(2)}$ Média da camada de $0-4 \mathrm{~cm} ;{ }^{(3)} \mathrm{CV}$ : coeficiente de variação do fator solo; ${ }^{(4)} \mathrm{CV}$ : coeficiente de variação do fator fonte/método. Médias seguidas de letras maiúsculas distintas, nas colunas e em cada tipo de solo, diferem significativamente entre si pelo teste de Tukey a $5 \%$. Médias seguidas de letras minúsculas distintas, nas linhas e em cada tipo de fonte e método, diferem significativamente entre si pelo teste de Tukey a $5 \%$.

\section{CONCLUSÕES}

1. Nas regiões com altas concentrações de $\mathrm{P}$, como as que ocorrem ao redor dos grânulos de adubos fosfatados, o P movimenta-se bem mais do que no restante da solução do solo, quando o movimento se restringe a poucos milímetros.

2. $\mathrm{O}$ efeito dos adubos fosfatados em aumentar o $\mathrm{P}$ varia com a distância dos grânulos e com o método de extração: junto aos grânulos, o SFT proporciona maiores valores de $\mathrm{P}$ do que o DAP; com o aumento da distância o efeito normalmente inverte.

3. A magnitude da variação na composição química do solo nas adjacências dos grânulos de fertilizantes em relação a pH, $\mathrm{Al}, \mathrm{Ca}$ e $\mathrm{Mg}$ difere com o tipo de solo e principalmente com o fosfato.

4. O método Mehlich-1 quantifica mais $\mathrm{P}$ do que o da resina trocadora de ânions nas proximidades das regiões fertilizadas com $\mathrm{P}$ e isso precisa ser considerado em estudo de calibração para fins de recomendação de adubação.

\section{LITERATURA CITADA}

AKINREMI, O.O. \& CHO, C.M. Phosphorus diffusion retardation in a calcareous system by coapplication of potassium chloride. Soil Sci. Soc. Am. J., 57:845-850, 1993.

BARBER, S.A. A diffusion and mass-flow concept of soil nutrient availability. Soil Sci., 93:39-49, 1962.

COMISSÃO DE QUÍMICA E FERTILIDADE DO SOLO - RS/ SC - CQFSRS/SC. Manual de adubação e calagem para os estados do Rio Grande do Sul e Santa Catarina. Porto Alegre, SBCS - Núcleo Regional Sul, 2004. 400p.

DIEROLF, T.S.; ARYA, L.M. \& YOST, R.S. Water and cation movement in an Indonesian Ultisol. Agron. J., 89:572$579,1997$.

ERNANI, P.R.; DIAS, J. \& FLORE, J.A. Annual additions of potassium to the soil increased apple yield in Brazil. Commun. Soil Sci. Plant Anal., 33:1291-1304, 2002.

ERNANI, P.R.; BAYER, C. \& RIBEIRO, M.F.S. Chemical modifications caused by liming below the limed layer in a predominantly variable charge acid soil. Commun. Soil Sci. Plant Anal., 35:889-901, 2004.

ERNANI, P.R. Química do solo e disponibilidade de micronutrientes. Lages, O autor, 2008. 230p.

GATIBONI, L.C.; KAMINSKI, J.; RHEINHEIMER, D.S. \& SAGGIN, A. Quantificação do fósforo disponível por extrações sucessivas com diferentes extratores em Latossolo Vermelho distroférrico. R. Bras. Ci. Solo, 26:1023-1029, 2002.

GATIBONI, L.C.; KAMINSKI, J.; RHEINHEIMER, D.S. \& BRUNETTO, G. Superphosphate and rock phosphates as phosphorus sources for grass-clover pasture on a limed acid soil in Southern Brazil. Commun. Soil Sci. Plant Anal., 34:2503-2514, 2003.

KELLY, S.F.; GREEN, J.L. \& SELKER, J.S. Fertilizer diffusion in container medium. J. Am. Soc. Hortic. Sci., 122:122128, 1997. 
MANTOVANI, A.; ERNANI, P.R. \& SANGOI, L. A adição de superfosfato triplo e a percolação de nitrogênio no solo. R. Bras. Ci. Solo, 31:887-895, 2007.

McKEAN, J. \& WARREN, G.P. Determination of phosphate desorption characteristics in soils using successive resin extractions. Commun. Soil Sci. Plant Anal., 27:2397-2417, 1996.

MURPHY, J. \& RILEY, J.P. A modified single solution method for the determination of phosphate in natural waters. Anal. Chim. Acta, 27:31-36, 1962.

NEVES, L.S.; ERNANI, P.R. \& SIMONETE, M.A. Mobilidade de potássio em solos decorrente da adição de doses de cloreto de potássio. R. Bras. Ci. Solo, 33:25-32, 2009.

OLIVEIRA, R.H.; ROSOLEM, C.A. \& TRIGUEIRO, R.M. Importância do fluxo de massa e difusão no suprimento de potássio ao algodoeiro como variável de água e potássio no solo. R. Bras. Ci. Solo, 28:439-445, 2004.
RHEINHEIMER, D.S.; ANGHINONI, I.; CONTE, E.; KAMINSKI, J. \& GATIBONI, L.C. Dessorção de fósforo avaliada por extrações sucessivas em amostras de solo provenientes dos sistemas plantio direto e convencional. Ci. Rural, 33:1053-1059, 2003.

SILVA, F.C. \& RAIJ, B.van. Disponibilidade de fósforo em solos avaliada por diferentes extratores. Pesq. Agropec. Bras., 34:267-288, 1999.

STEVEnson, F.J. \& COLE, M.A. Cycles of soil carbon, nitrogen, phosphorus, sulfur, micronutrients. 2.ed. New York, Wiley \& Sons, 1999. 427p.

TEDESCO, M.J.; GIANELLO, C.; BISSANI, C.A.; BOHNEN, H. \& VOLKWEISS, S.J. Análise de solo, plantas e outros materiais. Porto Alegre, UFRGS, 1995. 174p. 